\title{
Search for molecular emission from V838 Monocerotis
}

\author{
M. T. Rushton ${ }^{1}$, I. M. Coulson ${ }^{2}$, A. Evans ${ }^{1}$, L.-A. Nyman $^{3}$, B. Smalley ${ }^{1}$, T. R. Geballe ${ }^{4}$, J. Th. van Loon ${ }^{1}$, \\ S. P. S. Eyres ${ }^{5}$, and V. H. Tyne ${ }^{1}$ \\ 1 Astrophysics Group, School of Chemistry and Physics, Keele University, Staffordshire, ST5 5BG, UK \\ 2 Joint Astronomy Centre, 660 N. A 'ohōkū Place, University Park, Hilo, HI 96720, USA \\ ${ }^{3}$ European Southern Observatory, Alonso de Cordova 3107, Casilla 19001, Santiago 19, Chile \\ ${ }^{4}$ Gemini Observatory, 670 N. A'ohōkū Place, University Park, Hilo, HI 96720, USA \\ ${ }^{5}$ Centre for Astrophysics, University of Central Lancashire, Preston, Lancashire, PR1 2HE, UK
}

Received 21 February 2003 / Accepted 18 September 2003

\begin{abstract}
We present a search for ${ }^{12} \mathrm{CO}$ and $\mathrm{SiO}$ emission in V838 Mon. We derive upper limits on the ${ }^{12} \mathrm{CO}$ and $\mathrm{SiO}$ emission, and on the total mass of the circumstellar material. Using an excitation temperature of $1000 \mathrm{~K}$, and a likely distance of $6 \mathrm{kpc}$, the non-detection of ${ }^{12} \mathrm{CO}$ emission leads to a $3 \sigma$ upper limit on the mass of gas around the central star to be $10^{-2} M_{\odot}$.
\end{abstract}

Key words. stars: circumstellar matter - stars: individual: V838 Mon

\section{Introduction}

V838 Mon was discovered in outburst on 2002 January 6 by Brown (2002), and, initially, interpreted as a possible nova. A second, larger, outburst was detected on 2002 February 2, and a multi-maximum lightcurve developed over the months following discovery (Munari et al. 2002a). The initial spectra displayed a multitude of P Cygni profile spectral lines indicating mean outflows of $200-500 \mathrm{~km} \mathrm{~s}^{-1}$. These low velocities, coupled with the multi-peaked lightcurve and the lack of strong emission lines, rule out a classical nova interpretation. Furthermore, a light-echo, possibly indicating the existence of cirumstellar material, appeared on 2002 February 17 (Henden et al. 2002). Post-2002 February spectra revealed a cooling and expanding photosphere consistent with a heliumflash scenario (Raunch et al. 2002). However, the extreme luminosity of the 2002 February outburst, the speed of the evolution and, in particular, the oxygen-rich nature of V838 Mon (Evans et al. 2003), makes this model unlikely (Kimeswenger et al. 2002; Bond et al. 2002). Soker \& Tylenda (2003) showed that the complex lightcurve, both qualitatively and quantitatively, can be explained by the merging of two main-sequence stars. However, it has emerged that there is a possible B3 V component of V838 Mon (Munari et al. 2002b).

Near-infrared spectroscopy was reported by Geballe et al. (2002a), who noted the presence of deep ${ }^{12} \mathrm{CO}$ first overtone bands at $\sim 2.30 \mu \mathrm{m}$. Subsequent spectra showed a complex evolution of these ${ }^{12} \mathrm{CO}$ bands, initially involving peculiar $\Delta v=2$ bandheads, which seemed to display "extra absorption" in the R branch (Banerjee \& Ashok 2002). Later

Send offprint requests to: M. T. Rushton,

e-mail: mtr@astro.keele.ac.uk
Geballe et al. (2002b) noted these bandheads in a strange emission configuration on 2002 March 9, before returning to absorption by 2002 May 14 (Banerjee \& Ashok 2002). Such behaviour may be related to ${ }^{12} \mathrm{CO}$ in the light-echo material or, more likely, in the immediate stellar environment.

Here we report a search for molecular emission at submillimetre wavelengths in order to constrain the mass of gas in the circumstellar environment.

\section{Observations}

Heterodyne observations of the $\mathrm{SiO} J=2 \rightarrow 1(v=$ $86.2 \mathrm{GHz}),{ }^{12} \mathrm{CO} J=2 \rightarrow 1(v=230.5 \mathrm{GHz})$ and $J=1 \rightarrow$ $0(v=115.3 \mathrm{GHz})$ transitions of V838 Mon were taken at the Swedish ESO Submillimetre Telescope (SEST), using the High Resolution Spectrometer (HRS). Calibrations were performed by observing AH Sco, W Hya, VY CMa, IRSV1540 and RV Vel. Observations of the ${ }^{12} \mathrm{CO} J=3 \rightarrow 2$ transition $(v=345.8 \mathrm{GHz})$ were made at the James Clerk Maxwell Telescope (JCMT), using the B3 receiver. Further observing details are shown in Table 1. The diameter of the light-echo associated with V838 Mon had grown to 59" by 2002 August (Henden \& Munari 2002); given the beamwidths (Table 1), emission from the light-echo would be included in the beam, along with any from recent mass-loss.

The individual spectra were coadded and binned after a linear baseline was removed, with each bin corresponding to $2 \mathrm{~km} \mathrm{~s}^{-1}$. The spectra cover a range in velocity with respect to the local standard of rest, $V_{\mathrm{LSR}}$, from $-224.3 \mathrm{~km} \mathrm{~s}^{-1}$ to $+224.3 \mathrm{~km} \mathrm{~s}^{-1}\left({ }^{12} \mathrm{CO} J=3 \rightarrow 2\right),-654.4$ to $+653.5 \mathrm{~km} \mathrm{~s}^{-1}$ $\left({ }^{12} \mathrm{CO} J=2 \rightarrow 1\right),-110.8 \mathrm{~km} \mathrm{~s}^{-1}$ to $+110.8 \mathrm{~km} \mathrm{~s}^{-1}\left({ }^{12} \mathrm{CO} J=\right.$ $1 \rightarrow 0)$, and $-148 \mathrm{~km} \mathrm{~s}^{-1}$ to $+148 \mathrm{~km} \mathrm{~s}^{-1}(\mathrm{SiO} J=2 \rightarrow 1)$. 
Table 1. Observing details.

\begin{tabular}{cccccccc}
\hline \hline Transition & $\begin{array}{c}\text { Date } \\
(\mathrm{UT})\end{array}$ & Telecope & Efficiency & Beamwidth & $\begin{array}{c}\text { Integration time } \\
(\mathrm{s})\end{array}$ & $\begin{array}{c}\text { Resolution } \\
(\mathrm{kHz})\end{array}$ & $\begin{array}{c}T_{\text {sys }} \\
(\mathrm{K})\end{array}$ \\
\hline $\mathrm{SiO} J=2 \rightarrow 1$ & $05 / 03 / 03$ & SEST & 0.75 & $57^{\prime \prime}$ & 1440 & 42.6 & 147 \\
${ }^{12} \mathrm{CO} J=1 \rightarrow 0$ & $05 / 03 / 03$ & SEST & 0.7 & $45^{\prime \prime}$ & 1440 & 42.6 & 161 \\
${ }^{12} \mathrm{CO} J=2 \rightarrow 1$ & $07 / 03 / 03$ & SEST & 0.5 & $23^{\prime \prime}$ & 2040 & 699 & 373 \\
${ }^{12} \mathrm{CO} J=3 \rightarrow 2$ & $02 / 11 / 02$ & JCMT & 0.63 & $14^{\prime \prime}$ & 1800 & 62.5 & 323 \\
\hline
\end{tabular}
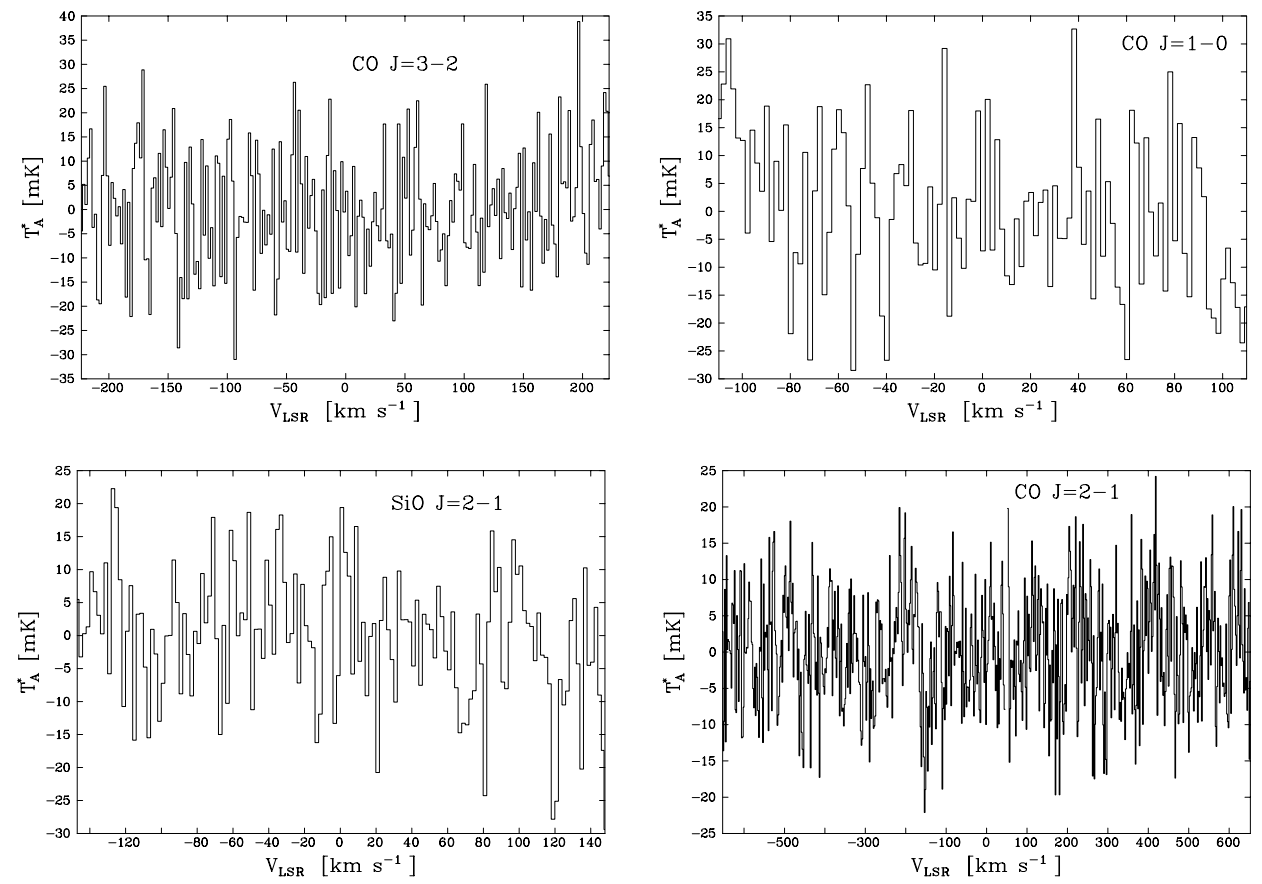

Fig. 1. Search for molecular emission in the region of ${ }^{12} \mathrm{CO} J=3 \rightarrow 2$ (upper left panel), ${ }^{12} \mathrm{CO} J=1 \rightarrow 0$ (upper right panel), ${ }^{12} \mathrm{CO} J=2 \rightarrow 1$ (lower right panel) and $\mathrm{SiO} J=2 \rightarrow 1$ (lower left panel) transitions in V838 Mon.

Given its possible distance (see Sect. 3), the $V_{\text {LSR }}$ of V838 Mon is expected to be $\sim 80 \mathrm{~km} \mathrm{~s}^{-1}$, assuming it is located in the galactic disk and that standard Galactic rotation applies; this lies well within our $V_{\mathrm{LSR}}$ ranges.

\section{Results and discussion}

The binned spectra are shown in Fig. 1; no obvious emission is evident. We obtain $3 \sigma$ upper limits on any molecular emission of $T_{A}^{*} \approx 40 \mathrm{mK}\left({ }^{12} \mathrm{CO} J=1 \rightarrow 0 ;{ }^{12} \mathrm{CO} J=3 \rightarrow 2\right)$, $T_{A}^{*} \approx 25 \mathrm{mK}\left({ }^{12} \mathrm{CO} J=2 \rightarrow 1\right)$ and $T_{A}^{*} \approx 30 \mathrm{mK}$ ( $\mathrm{SiO} J=2 \rightarrow 1$ ), with a resolution of $2 \mathrm{~km} \mathrm{~s}^{-1}$, and a corresponding $3 \sigma$ upper limit of $1 \mathrm{~K} \mathrm{~km} \mathrm{~s}^{-1}\left({ }^{12} \mathrm{CO} J=1 \rightarrow 0\right.$; $\left.{ }^{12} \mathrm{CO} J=3 \rightarrow 2\right), 0.63 \mathrm{~K} \mathrm{~km} \mathrm{~s}^{-1}\left({ }^{12} \mathrm{CO} J=2 \rightarrow 1\right)$ and $0.75 \mathrm{~K} \mathrm{~km} \mathrm{~s}^{-1}$ ( $\mathrm{SiO} J=2 \rightarrow 1$ ) for the integrated line flux over velocity intervals of $50 \mathrm{~km} \mathrm{~s}^{-1}$. The mass (in $\mathrm{kg}$ ) of a given molecular species within the beam is given by:

$$
\begin{aligned}
M_{\mathrm{mol}}= & \frac{8 \pi k^{2} m_{\mathrm{H}} D^{2} v^{2} \mathrm{~d} \Omega M T_{\text {rot }}}{h^{2} c^{3} A \Gamma(2 J+1) B} \\
& \times \exp \left\{\frac{h B J(J+1)}{k T_{\text {rot }}}\right\} \int T_{A}^{*} \mathrm{~d} V
\end{aligned}
$$

where $m_{\mathrm{H}}$ is the mass of a hydrogen atom, $M$ is the molecular weight of the molecule, $B$ is the rotational constant, $A$ is the Einstein Coefficient for the transition (Chandra et al. 1996 for ${ }^{12} \mathrm{CO}$; Lambert \& Vanden Bout 1978 for $\mathrm{SiO}$ ); the efficiency and beamwidth of the telescope are, respectively, $\Gamma$ and $\mathrm{d} \Omega . T_{\text {rot }}$ is the excitation temperature, and $D$ is the distance. The latter parameter is not well constrained for V838 Mon: Munari et al. (2002a) derive 780 pc, Kimeswenger et al. (2002) obtained $650 \mathrm{pc}$ and Bond et al. (2003) derive a lower limit of $6 \mathrm{kpc}$; we adopt $6 \mathrm{kpc}$ here.

The most likely source of molecular emission would be from the mass-loss associated with the recent outbursts. However, we cannot rule out the possibility of emission from the light-echo material. Therefore we consider excitation temperatures typical of diffuse interstellar material (light-echo) and circumstellar environments. In adopting these values we have chosen to neglect the possible B-star companion of V838 Mon, since its exact nature is unclear and it may just be a field star. The excitation temperature in the ISM, as deduced from ${ }^{12} \mathrm{CO} J=1 \rightarrow 0$, is $\sim 10 \mathrm{~K}$ (Staguhn et al. 1997), and the kinetic temperature is $\sim 77 \mathrm{~K}$ (Reach et al. 1994). For circumstellar material, the depth of the molecular bands has suggested 
Table 2. The $3 \sigma$ upper limits on the mass of a given molecular species and the corresponding total mass of gas in the beam within the circumstellar envelope around V838 Mon for an assumed distance of $6 \mathrm{kpc}$.

\begin{tabular}{ccccccc}
\hline \hline & \multicolumn{3}{c}{ Upper limits on mass of molecular species $\left(M_{\odot}\right)$} & \multicolumn{3}{c}{ Upper limits on total mass of gas $\left(M_{\odot}\right)$} \\
& $10 \mathrm{~K}$ & $100 \mathrm{~K}$ & $1000 \mathrm{~K}$ & $10 \mathrm{~K}$ & $100 \mathrm{~K}$ & $1000 \mathrm{~K}$ \\
\hline${ }^{12} \mathrm{CO} J=1 \rightarrow 0$ & $3.9 \times 10^{-5}$ & $2.2 \times 10^{-6}$ & $6.4 \times 10^{-6}$ & $1.3 \times 10^{-1}$ & $7.3 \times 10^{-3}$ & $2.1 \times 10^{-2}$ \\
${ }^{12} \mathrm{CO} J=2 \rightarrow 1$ & - & $1.6 \times 10^{-7}$ & $2.7 \times 10^{-7}$ & - & $5.2 \times 10^{-4}$ & $9.0 \times 10^{-4}$ \\
${ }^{12} \mathrm{CO} J=3 \rightarrow 2$ & - & $3.5 \times 10^{-7}$ & $1.1 \times 10^{-7}$ & - & $1.2 \times 10^{-3}$ & $3.5 \times 10^{-4}$ \\
$\mathrm{SiO} J=2 \rightarrow 1$ & - & $2.2 \times 10^{-2}$ & $4.4 \times 10^{-9}$ & - & $3.3 \times 10^{3}$ & $6.7 \times 10^{-4}$ \\
\hline
\end{tabular}

a temperature $\lesssim 1300 \mathrm{~K}$ (Evans et al. 2003). Therefore we consider three excitation temperatures: 10, 100 and 1000 K. From these values we thus obtain $3 \sigma$ upper limits on the mass associated with a given molecule in the beam (Table 2). Further, using the $\mathrm{H}_{2}$-to- $\mathrm{CO}$ and $\mathrm{H}_{2}$-to-SiO mass ratio for a circumstellar envelope around an O-rich star, $3.0 \times 10^{-4}$ and $9.8 \times 10^{-6}$ respectively (Bujarrabal et al. 1994), we can constrain a $3 \sigma$ upper limit on the total mass of gas (Table 2). This would lead us to underestimate the total mass of gas if V838 Mon was hydrogen deficient. However, this is precluded by the presence of strong $\mathrm{H}_{2} \mathrm{O}$ bands, which have been observed in the nearinfrared (Evans et al. 2003).

We note that Banerjee \& Ashok (2002) constrained the mass ejected from the recent eruption events on the basis of Ti I emission lines for a distance of $790 \mathrm{pc}$, and $T_{\text {rot }}=2600$ to $5000 \mathrm{~K}$; they determined this mass to lie between $10^{-7}$ to $10^{-5} M_{\odot}$. Scaling these values for a distance of $6 \mathrm{kpc}, 10^{-5}$ to $10^{-3} M_{\odot}$ is obtained, which is in the range suggested by our results (Table 2). However, the outburst related ejecta may be expanding at velocities in excess of the velocity ranges in which we have observed here, in which case any associated molecular emission would have not been detected in our study. However, this possibility can be ruled out for the ${ }^{12} \mathrm{CO} J=$ $2 \rightarrow 1$ data, which spans the likely range of outflow velocities (see Sect. 1).

Munari et al. (2002a) suggest that the light-echo may be associated with material lost by an Asymptotic Giant Branch (AGB) progenitor. Assuming the typical values of mass-loss rate, duration and velocity for an AGB star (Habing 1996), then the beam could contain $10^{-3}$ to $10^{-2} M_{\odot}$. Therefore it is clear from Table 2 that our values suggest that it is possible that, had we detected any molecular emission, it might have originated in the light-echo material. It must be noted that given the beamwidths relative to the diameter of the light-echo any extended ${ }^{12} \mathrm{CO}$ emission would have been missed.

\section{Conclusions}

A $3 \sigma$ upper limit on the emission of ${ }^{12} \mathrm{CO}$ and $\mathrm{SiO}$ from V838 Mon has been measured. We have further obtained upper limits on the mass comprised by these molecules and the corresponding total mass of gas in the circumstellar environment. For a possible distance of $6 \mathrm{kpc}$, we deduce a $3 \sigma$ upper limit on the total mass of gas in the circumstellar environment out to a distance $\lesssim 1 \mathrm{pc}$ from the object to be of the order of $10^{-4} M_{\odot}$ if expelled during the recent eruption, to $10^{-3} M_{\odot}$ if lost prior to the eruption.

It would be useful if future monitoring could be undertaken. In addition, a determination of the $V_{\mathrm{LSR}}$ of V838 Mon would prove helpful, along with further distance measurements.

Acknowledgements. M.T.R. is supported by a Particle Physics and Astronomy Research Council (PPARC) studentship. T.R.G. is supported by the Gemini Observatory, which is operated by the Association of Universities for Research in Astronomy, Inc., on behalf of the international Gemini partnership of Argentina, Australia, Brazil, Canada, Chile, the United Kingdom, and the United States of America. The JCMT is operated by the Joint Astronomy Centre on behalf of PPARC, the Netherlands Organization for Scientific Research and the National Research Council of Canada. The SwedishESO Submillimeter Telescope is operated by the Swedish National Facility for Radio Astronomy, Onsala Space Observatory at Chalmers University of Technology, and by ESO. Data reduction was carried out using hardware and software provided by PPARC.

\section{References}

Banerjee, D. P. K., \& Ashok, N. M. 2002, A\&A, 395, 161

Bond, H. E., Panagia, N., Sparks, B. W., Starrfield, S. G., \& Wagner, R. M. 2002, IAUC, 7892

Bond, H. E., Henden, A., Levay, Z. G., et al. 2003, Nature, 422, 405

Brown, N. J. 2002, IAUC, 7785

Bujarrabal, V., Fuente, A., \& Omont, A. 1994, A\&A, 285, 247

Chandra, S., Maheshwari, V. U., \& Sharma, A. K. 1996, A\&AS, 117, 557

Evans, A., Geballe, T. R., Rushton, M. T., et al. 2003, MNRAS, 343, 1054

Geballe, T. R., Eyres, S. P. S., Evans, A., \& Tyne, V. H. 2002a, IAUC, 7796

Geballe, T. R., Evans, A., Smalley, B., Tyne, V. H., \& Eyres, S. P. S. 2002b, IAUC, 7855

Habing, H. J. 1996, A\&ARv, 7, 97

Henden, A., Munari, U., \& Schwartz, M. 2002, IAUC, 7859

Henden, A., \& Munari, U. 2002, IAUC, 7958

Kimeswenger, S., Lederle, C., Schmeja, S., \& Armsdorfer, B. 2002, MNRAS, 336, 43

Lambert, D. L., \& Vanden Bout, P. A. 1978, ApJ, 221, 854

Munari, U., Henden, A., Kiyota, S., et al. 2002a, A\&A, 389, L51

Munari, U., Desidera, S., \& Henden, A. 2002b, IAUC, 8005

Raunch, T., Kerber, F., \& van Wyk, F. 2002, IAUC, 7886

Reach, W. T., Koo, B.-C., \& Heiles, C. 1994, ApJ, 429, 672

Soker, N., \& Tylenda, R. 2003, ApJ, 582, 105

Staguhn, J., Stutzki, J., Chamberlin, R. A., et al. 1997, ApJ, 491, 191 